\title{
Integridad científica: necesidad que la normativa nacional incluya criterios para decidir ante casos de mala conducta científica
}

\author{
Scientific integrity: there is a need for national \\ regulations to include assessment criteria in cases of \\ scientific misconduct
}

Correspondencia Ciro Maguiña Vargas ciro.maguina@upch.pe

Recibido: 10/06/2018 Aprobado: 13/06/2018

Citar como: Maguiña Vargas C. Integridad científica: necesidad que la normativa nacional incluya criterios para decidir ante casos de mala conducta cientifica [editorial]. Acta Med Peru. 2018;35(2):85-6

\section{Ciro Maguiña Vargas ${ }^{1,2, a}$}

1 Departamento de Enfermedades Infecciosas Tropicales y Dermatológicas, Hospital Cayetano Heredia. Lima, Perú.

2 Facultad de Medicina Alberto Hurtado, Universidad Peruana Cayetano Heredia. Lima, Perú.

a Infectológo tropicalista y dermatólogo

Hoy en día la investigación científica requiere no solo cumplir con las recomendaciones dadas por diversas normas nacionales e internacionales en las que están implícitos los aspectos éticos. En el año 1981, en los Estados Unidos, se divulgaron cuatro casos de mala conducta científica (MCC), hecho que llamó la atención del Congreso de los Estados Unidos de Norteamérica; posteriormente, fue más frecuente la aparición de casos similares (plagio, fraude científico, etc.). Ante esto, en el año 1989 se creó en Estados Unidos una comisión de expertos encargada de analizar este tipo de problemas; en 1992, se creó la Oficina de Integridad Científica (ORI) y en 1995 la Comisión Ryan presentó un reporte que detallaba las definiciones de integridad científica (IC), mala conducta en ciencia, etc. ${ }^{[1]}$ En el 2010 se crearon guías y documentos que promovían las recomendaciones dadas por la ORI.

La preocupación por la MCC generó múltiples reacciones. En el año 2007 se desarrolló en Lisboa la primera Conferencia Internacional sobre IC, en el 2010 la importante y trascendente $2^{\circ}$ Conferencia Internacional de Integridad en la Investigación y la Declaración de Singapur; en el 2013 la $3^{\circ}$ Conferencia Internacional sobre Integridad Científica realizada en Montreal, Canadá.

En las últimas décadas, en los diversos medios de comunicación, se han denunciado a muchas personalidades, escritores, políticos, científicos y otros por delitos de plagio o fraude científico; en las elecciones presidenciales del año 2016 un candidato a la presidencia fue denunciado por un presunto plagio en su tesis doctoral; a fines del 2017 un médico argentino solicitó se investigue el supuesto plagio de su libro "Relato oficial: lesión quirúrgica de la vía biliar", publicado en el 2017 por un médico peruano a través del Fondo Editorial Comunicacional (FEC) del Colegio Médico del Perú (CMP) ${ }^{[2]}$ del cual formé parte. Al respecto, en el CMP tanto en el reglamento como en el estatuto actuales no existen los conceptos modernos de integridad científica y mala conducta científica; tampoco en el Código de Ética vigente ${ }^{[3]}$.

Este último, sobre el trabajo de investigación, en el artículo 42 del capítulo 6 dice: "Todo médico que investiga debe hacerlo respetando la normativa internacional y nacional que regula la investigación con seres humanos, tales como las "Buenas Prácticas Clínicas", la Declaración de Helsinki, la Conferencia Internacional de Armonización, el Consejo Internacional de Organizaciones de las ciencias Médicas (CIOMS) y el Reglamento de Ensayos clínicos del Ministerio de Salud" y en el art. $48^{\circ}$ : "El médico debe presentar la información proveniente de una investigación médica, para su publicación, independientemente de los resultados, sin incurrir en falsificación ni plagio y declarando si tiene o no conflicto de interés" [3].

Es por ello que el Comité Editorial del FEC, en enero del 2018, creyó conveniente proponer al CMP, a través de la aprobación del Comité de Doctrina y Legislación del CMP, que se considere incluir estos conceptos modernos dentro de la normatividad, a fin de que cuando haya acusaciones a los colegas sobre MCC se tengan acuñados estos conceptos. Otro hecho importante es que en gran parte de las universidades peruanas y otras instituciones, como es el CMP, no existen sistemas modernos informáticos antiplagio de manera obligatoria y formal.

Felizmente la ciencia ha definido claramente los conceptos de IC y MCC, los cuales están normados de manera importante a nivel internacional; pero en el Perú estos conceptos todavía están muy atrasados y lamentablemente solo una Universidad tiene normado y reglamentado el tema desde el 2017 de manera obligatoria, tanto para los estudiantes de pregrado, posgrado y los docentes ${ }^{[4]}$. 
Se define a la integridad científica como el marco de valores que permiten asegurar que la investigación se conduce de manera honesta y precisa; la palabra integridad, deriva del latín integrîtas: integro, intacto, probo, honesto ${ }^{[5]}$.

Existe una conducta responsable en investigación cuando la forma de actuar del investigador es acorde con los principios y valores de la ciencia; cuando se desarrolla, propone y/o evalúa un reporte de investigación, esta se hará de manera honesta y precisa ${ }^{\left[{ }^{[6]}\right.}$.

Se entiende por mala conducta o práctica científica a aquella acción relacionada con la fabricación, falsificación o plagio en la propuesta, realización o revisión de investigaciones o en la publicación de sus resultados, esta mala práctica es cometida deliberadamente o intencionalmente; no se incluyen los errores honestos o las diferencias de opinión ${ }^{[7,8]}$.

Algunos tipos de MCC son ${ }^{[8-11]}$ : 1) fabricación: el acto de inventar datos o resultados y registrarlos o reportarlos como propios; 2) falsificación: la manipulación de datos o resultados, el cambio u omisión de tal manera que la investigación no esté representada con exactitud en el documento publicado; 3) plagio: la acción de apropiarse de las ideas, procesos, resultados o palabras de otros sin dar el crédito o reconocimiento correspondiente, se presenta de muchas formas y es la MCC más común; y 4) autoplagio: una variante del plagio que sucede cuando uno mismo se copia.

Además, debemos referirnos a otras denominadas prácticas cuestionables en investigación, entre las que se encuentran: 1) mala representación: cuando no se identifica correctamente la contribución de investigador en su publicación, cuando hay cambio en la lista de autores en las publicaciones, cuando se citan publicaciones que han sido rechazadas indicando que están "en proceso" o realizar la publicación de la misma información en diferentes medios (publicación duplicada, triplicada, etc.); 2) inexactitud: cuando existen errores al citar referencias, errores al referirse al contenido de las citas, no resumir adecuadamente los hallazgos o conclusiones, no dar suficiente información sobre los métodos, reportar mal los análisis estadísticos o usar inapropiadamente las pruebas estadísticas al analizar sus datos.

En cuanto a la frecuencia de MCC existen numerosos trabajos en el mundo. Titus et al. encuestaron 2212 investigadores biomédicos y encontraron que el $8,7 \%$ indicaba haber observado o tenido una prueba directa de mala conducta en investigación durante los últimos tres años, el 60\% de los casos de MCC correspondía a falsificación o fabricación y $36 \%$ a plagio; el $37 \%$ de los incidentes no había sido reportado ${ }^{[12]}$. Fanelli, mediante una revisión sistemática, analizó a 21 encuestas realizadas a miles de investigadores acerca de MCC, casi el $2 \%$ de los entrevistados admitió haber fabricado o falsificado datos o resultados al menos una vez, el 33,7\% admitió haber realizado otras prácticas de investigación cuestionables ${ }^{[13]}$. Martinson et al. estudiaron a 3247 científicos de Estados Unidos y encontraron que un 1,4\% usaba las ideas de otros sin obtener permiso o sin darles su crédito merecido, 4,7\% publicó los mismos datos o resultados en dos a más revistas, $33 \%$ admitió haber realizado otra forma de mala conducta en investigación ${ }^{[14]}$.

En el Perú, existen pocos estudios sobre estos temas. En el año 2010, Saldaña et al. indicaron que existía una alta frecuencia de plagio en tesis para obtener el grado de bachiller en la carrera de medicina humana pertenecientes a una universidad pública peruana, concluyeron que “... se encontró evidencia de plagio en $27 / 33$ de las introducciones, 37,3\% (171/479) de todos los párrafos analizados tuvieron algún grado de plagio, el plagio literal fue el más frecuente (20/27) y las fuentes de plagio más comunes fueron revistas científicas (19/27). Las características de los estudios analizados fueron observacionales (32/33), transversales (30/33), descriptivos (25/33) y retrospectivos (19/33)..." [15].

La Declaración de Singapur sobre la Integridad en la Investigación fue elaborada en el marco de la $2^{\circ}$ Conferencia Mundial sobre Integridad en la Investigación, con el fin de servir como una guía global para la conducta responsable en la investigación; a continuación presentamos los principios descritos en dicha Declaración ${ }^{[16]}$ :

1) Honestidad en todos los aspectos de la investigación, 2) responsabilidad en la ejecución de la investigación, 3) cortesía profesional e imparcialidad en las relaciones laborales y 4) buena gestión de la investigación en nombre de otros.

\section{REFERENCIAS BIBLIOGRÁFICAS}

1. Davis MS, Riske-Morris M, Diaz SR. Causal factors implicated in research misconduct: Evidence from ORI case files. Sci Eng Ethics. 2007;13(4):395-414.

2. Sociedad de Cirujanos Generales del Perú. Comunicado. Boletín Electrónico [Internet]. 2018 [citado el 15 de junio de 2018];14(29). Disponible en: http://www.scgp.org/boletin-cirujanos-scgp/pdf/2018-FEBRERO.pdf

3. Colegio Médico del Perú. Código de Ética y Deontología [Internet]. Lima: Colegio Médico del Perú; 2007 [citado el 15 de junio de 2018]. Disponible en: http://medicina.unmsm.edu.pe/etica/images/ Postgrado/Instituto_Etica/Codigo_etica_cmp_OCT-2007.pdf

4. Universidad Peruana Cayetano Heredia, Vicerrectorado de Investigación. Políticas para responder alegaciones de mala conducta científica. Lima: Universidad Peruana Cayetano Heredia; 2017.

5. Guarino J. Conducta responsable en investigación. Tacna: Universidad Privada de Tacna; 2016.

6. Garcia P. Introducción a la conducta responsable en investigación (CRI). Lima: Andean Quipu, Universidad Peruana Cayetano Heredia; 2013.

7. Iverson C. AMA manual of style: A guide for authors and editors. 10th ed. New York, NY: Oxford University Press Inc.; 2007.

8. PoliScience. La publicación científica [Internet]. Valencia: Universitat Politècnica de València; 2012 [citado el 15 de junio de 2018]. Disponible en: http://poliscience.blogs.upv.es/investigadores-2/publicar/
9. Pamo O. Fraude en el mundo científico. Acta Herediana. 2007;42:17-31.

10. Barranguet C. Cómo producir un artículo para una revista internacional. New York: Elsevier; 2015.

11. Silva $\mathrm{D}$, Llanes $\mathrm{R}$, Rodríguez $\mathrm{A}$. Manifestaciones impropias en la publicación científica. Rev Cubana Salud Pública [Internet]. 2007 [citado el 15 de junio de 2018];33(4). Disponible en: http://bvs.sld. cu/revistas/spu/vol33_4_07/spu09407.html

12. Titus SL, Wells JA, Rhoades $\mathrm{L}$. Repairing research integrity. Nature. 2008;453(7198):980-2.

13. Fanelli $D$. How many scientists fabricate and falsify research? A systematic review and meta-analysis of survey data. PLoS ONE. 2009;4(5):e5738.

14. Martinson B, Anderson $M$, de Vries R. Scientists behaving badly. Nature. 2005;435(7043):737-8.

15. Saldaña-Gastulo JJC, Quezada-Osoria CC, Peña-Oscuvilca A, MaytaTristán P. Alta frecuencia de plagio en tesis de medicina de una universidad pública peruana. Rev Peru Med Exp Salud Publica. 2010;27(1):63-7.

16. Fondo Nacional de Desarrollo Científico y Tecnológico. Declaración de Singapur sobre la Integridad en la Investigación [Internet]. Santiago de Chile: Comisión Nacional de Investigación Científica y Tecnológica; 2016 [citado el 15 de junio de 2018]. Disponible en: http://www.conicyt.cl/ fondap/files/2014/12/DECLARACI\%C3\%93N-SINGAPUR.pdf 